STRUCTURAL BIOLOGY

ISSN 2059-7983

Received 24 March 2019

Accepted 23 July 2019

₹ These authors made equal contributions.

Keywords: cryo-EM; merging of data; singleparticle processing; RELION.

Supporting information: this article has supporting information at journals.iucr.org/d

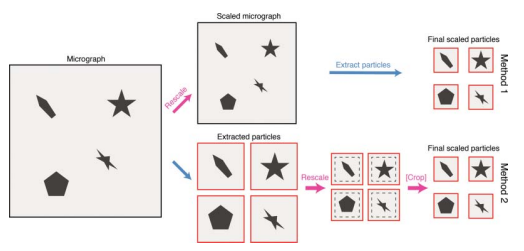

OPEN $\odot$ ACCESS

\section{Methods for merging data sets in electron cryo-microscopy}

\author{
Max E. Wilkinson,‡ Ananthanarayanan Kumar‡ and Ana Casañal*
}

MRC Laboratory of Molecular Biology, Francis Crick Avenue, Cambridge Biomedical Campus, Cambridge CB2 OQH, England. *Correspondence e-mail: acasanal@mrc-Imb.cam.ac.uk

Recent developments have resulted in electron cryo-microscopy (cryo-EM) becoming a useful tool for the structure determination of biological macromolecules. For samples containing inherent flexibility, heterogeneity or preferred orientation, the collection of extensive cryo-EM data using several conditions and microscopes is often required. In such a scenario, merging cryoEM data sets is advantageous because it allows improved three-dimensional reconstructions to be obtained. Since data sets are not always collected with the same pixel size, merging data can be challenging. Here, two methods to combine cryo-EM data are described. Both involve the calculation of a rescaling factor from independent data sets. The effects of errors in the scaling factor on the results of data merging are also estimated. The methods described here provide a guideline for cryo-EM users who wish to combine data sets from the same type of microscope and detector.

\section{Introduction}

Over the last decade, electron cryo-microscopy (cryo-EM) has become a powerful tool to resolve three-dimensional (3D) structures of biological specimens at a resolution sufficient for proposing de novo atomic models (Kühlbrandt, 2014; Smith \& Rubinstein, 2014; Cheng et al., 2017). This has primarily been possible through progress made in the development of direct electron detectors (Battaglia et al., 2009; Faruqi \& McMullan, 2011; Li et al., 2013; McMullan et al., 2014) and improvements in image-processing algorithms (Scheres \& Carazo, 2009; Scheres, 2012). The result of these advances is a rapid growth in the number of cryo-EM structures deposited per year in the Electron Microscopy Database (EMDB; https://www.ebi.ac.uk/ pdbe/emdb/statistics_mol_wt.html/, https://www.ebi.ac.uk/pdbe/ emdb/statistics_min_res.html/).

Cryo-EM can be performed on small amounts of biological samples (in the microlitre to femtolitre range; Russo \& Passmore, 2016; Ashtiani et al., 2018; Noble, Wei et al., 2018) in a relatively short time (from hours to days; Kimanius et al., 2016; Cianfrocco et al., 2018). A suitable sample for cryo-EM is homogenous and evenly dispersed in random orientations throughout the support holes. However, many biological specimens are challenging targets that possess intrinsic flexibility, conformational heterogeneity or adopt a preferred orientation in the vitreous layer of ice (Nogales et al., 2016; Plaschka et al., 2017). Such problems can be reduced by optimizing the biochemical preparation of the sample, changing the type and chemistry of the support grids (Meyerson et al., 2014; Russo \& Passmore, 2014; Boland et al., 2017; Liu et al., 2019) and using different detergents (Takizawa et al., 2017; Drulyte et al., 2018; Chen et al., 2019). All of the above can 
lead to the necessity to collect data in various conditions. For low-concentration samples, or when inherent heterogeneity or preferred particle orientation is difficult to eliminate, collecting a large amount of cryo-EM data is a valid option. For example, the number of particles with rare views might be enriched in large data sets, as distribution and orientation can highly depend on the ice thickness (Casañal et al., 2017; Noble, Dandey et al., 2018). Also, large data sets can allow extensive classification to computationally isolate subpopulations of particles in different states (Fernández et al., 2013; Urnavicius et al., 2015; Matzov et al., 2017; Wilkinson et al., 2018; Charenton et al., 2019; Guo et al., 2019).

There are many practical limitations when it comes to collecting and processing such large cryo-EM data sets. The lack of availability of ample microscope time within an institute or cryo-EM facility means that users often collect several data sets with different microscopes, either within their host institute or across the world in dedicated cryo-EM facilities (Casañal et al., 2017; Menny et al., 2018; Fica et al., 2019). Also, when microscopes are under repair or upgraded (for example the incorporation of a new detector or energy filter), or when changing the magnification for data collection is needed (for example increasing the resolution or the number of particles per image), users might collect at different pixel sizes. The absence of a streamlined procedure to combine cryo-EM data that differ in pixel size poses an additional challenge in structure determination in single-particle projects. Here, we describe two straightforward methods to combine cryo-EM data sets (Figs. 1 and 2). We present two case studies. In the first, data sets were collected using two distinct Titan Krios microscopes with similar magnifications. In the second, data acquisition was performed on the same Titan Krios microscope at different magnifications.

\section{Procedure}

\subsection{General considerations}

Before combining data sets, it is essential to take into account two primary considerations. Firstly, the relative pixel size ( $\AA$ per pixel) between data sets needs to be accurately determined. One data set must be rescaled to match the other (the reference) such that the particles can be aligned to the same 3D reference. The success of this process will depend on how accurately the relative pixel sizes are estimated. Secondly, the absolute pixel size of the reference data set should be determined precisely. Accurate knowledge of the absolute pixel size is required for defocus determination, contrast transfer function (CTF) correction and map interpretation (Wade, 1992; Cheng et al., 2015). The exact absolute pixel size often deviates from the nominal pixel size owing to slight differences in the optics and position of the detectors between different microscopes of the same type. Ideally, one could determine the absolute pixel size for both data sets before or during data collection. This can be performed using methods such as cross-grating grids (lower magnification regime), titanium dioxide (medium magnification regime) and gold on grids in the form of gold particles or gold foil (higher magnification regime) (Cheng et al., 2015). UltraAufoil grids are particularly useful as the reflections of the gold on the grid can be used to calibrate the pixel size under the same imaging conditions as used for data collection (Russo \& Passmore, 2014; Cheng et al., 2015). If pixel sizes are accurately determined before data processing one can rescale the different sets of micrographs to have the same pixel size and treat the data sets as one.

However, in many cases users rely on the nominal pixel size given by the facility and might not be able to determine the

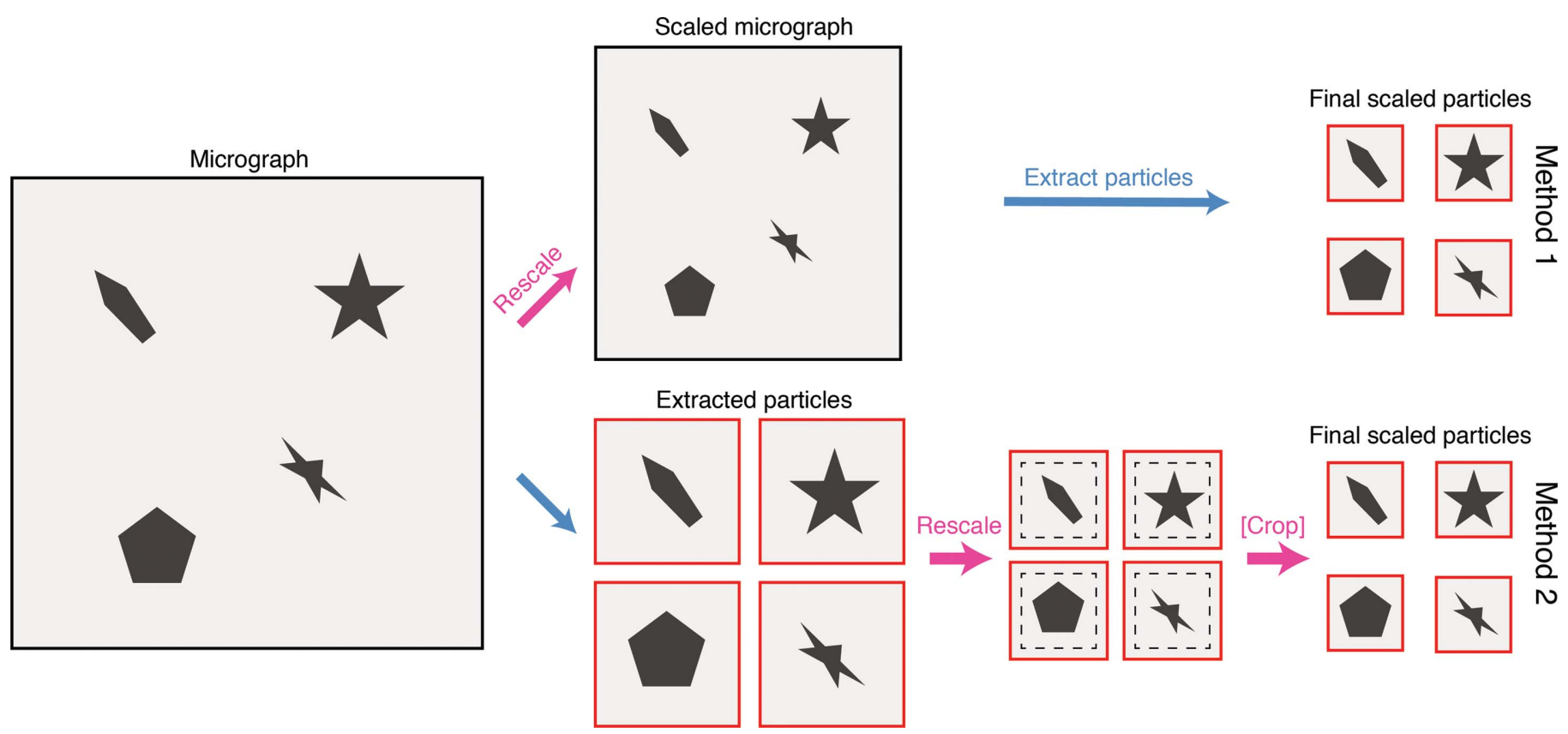

Figure 1

Schematic overview of two methods for scaling cryo-EM data. In Method 1, micrographs are scaled (pink arrow) before particles are extracted (blue arrow). In Method 2, particles are extracted and then scaled, with a optional cropping step if required. 


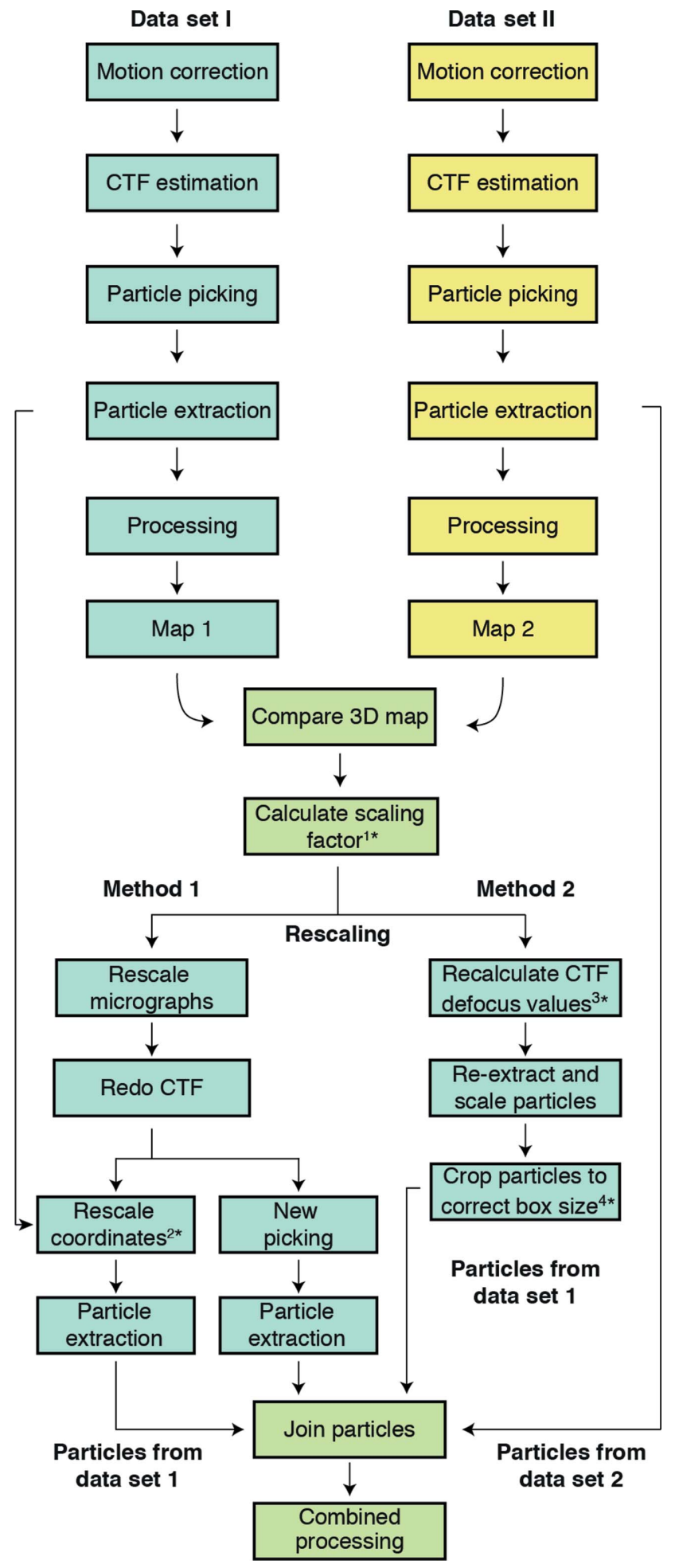

Figure 2

Flowchart of the data-merging process. The first step for both methods is obtaining independent $3 \mathrm{D}$ reconstructions to calculate the scaling factor between data sets. Method 1 rescales the data at the level of micrographs and Method 2 uses extracted particles. It is essential to redo the CTF estimation (Method 1) or to apply the scaling factor to defocus values to recalculate the CTF (Method 2). Once the particles from the two data sets have been merged ('Join' job in RELION), further processing can be carried out using standard procedures. Asterisks $(*)$ indicate where scripts are provided to perform different steps in the process: 1*, determine_relative_pixel_size.py; 2*, rescale_ particles.py; $3^{*}$, scale_ctf.sh; 4*, boxscaler.py. exact pixel size after their microscope session has ended, or the absolute pixel sizes might have been measured inaccurately. For this, we present an empirical approach to determine the pixel size of one data set relative to the nominal pixel size of the reference data set. Even if the exact pixel size is known, this method can be used to confirm the relative pixel size between data sets.

\subsection{Determination of the scaling factor between data sets by} cross-correlation

In principle, determination of the scaling factor between data sets by cross-correlation is straightforward. All that is needed are independent 3D reconstructions for each data set. By cross-correlating the maps at different relative pixel sizes it is possible to determine their scaling factor. To illustrate this concept (and later demonstrate Method 1; Section 2.3.1), two different data sets of the polymerase module of the cleavage and polyadenylation factor (CPF) from Saccharomyces cerevisiae are used, collected at nominal pixel sizes of $1.40 \AA$ per pixel (data set I, Krios 2 at MRC-LMB) and $1.36 \AA$ per pixel [data set II, Diamond Light Source (DLS) electron BioImaging Centre (eBIC)] (Casañal et al., 2017). To calculate the relative pixel size and scale the data, the pixel size of the data set with the larger pixel size (1.40 A per pixel; reference; data set $I$ in this example) should be kept constant, while changing the relative pixel size of the second data set (1.36 ̊ per pixel; data set II).

2.2.1. Obtain 3D reconstructions from each data set. In RELION, the processing of individual data sets involves motion correction, CTF estimation, particle picking, extraction, several classification steps and refinement (Scheres, 2012; Fernandez-Leiro \& Scheres, 2017). More extensive processing can be performed, including Bayesian polishing and CTF refinement (Zivanov et al., 2018, 2019). However, these additional steps are not usually required before rescaling data sets (Fig. 2). Nevertheless, it is desirable to obtain high-resolution $3 \mathrm{D}$ reconstructions of individual data sets, as the resolution of the maps is directly linked to the accuracy of the scaling factor. In most cases, $4-5 \AA$ resolution is sufficient. If large flexible areas are present, the comparison of maps can be improved by masking.

2.2.2. Calculate and optimize the scaling factor between 3D maps. Real-space correlation is recommended to compare the volumes of independently calculated maps. Crosscorrelation in real space can be determined, for example, using Chimera (Pettersen et al., 2004), where superposition and fitting of the maps can be achieved using the 'Fit in Map' command [Fig. 3(a)]. This tool provides a correlation coefficient between the maps that indicates the quality of the fitting. The Volume Viewer tool can be used to adjust the pixel size, using real-space interpolation, of the target map [data set II, map 2, Fig. 3(a)] by entering an adjusted voxel size into 'Features', 'Coordinates'.

Also, we provide a Python script (https://www.python.org) called determine_relative_pixel_size.py (Supplementary Script 1) that uses relion_image_handler (Scheres, 
2012) and the Fourier shell correlation (FSC; Harauz \& van Heel, 1986) to determine an optimal pixel size for the target map. The output is a pixel size range in which the FSC remains the same. This script uses the files map1.mrc and map2. mrc and their corresponding pixel sizes as input, carries out rescaling of map 2 to a set of pixel sizes and measures crosscorrelation between the two 3D volumes of interest by FSC. The script can be run by typing the following text in the command line:

python determine_relative_pixel_size.py -ref_map mapl.mrc --angpix_ref_map 1.40 --map map2.mrc--angpix_map_nominal 1.36

The result appears as follows:

BEST: 1.282 range: $1.274-1.29$

The precision that can be obtained depends on several factors. If one uses the script, and therefore relion_image_ handler, one limitation is the rescaling, since relion_image handler uses padding/cropping in reciprocal space. This is dependent on the box size, which means that the accuracy is inversely dependent on the box size. For a 100-pixel box size, the precision will be $2 \%$ of the pixel size $(0.02 \AA$ for a pixel size of $1 \AA$ per pixel). With a 200-pixel box size it will be $1 \%$ of the pixel size. In our tests, we observed that the crosscorrelation shows a finer sampling using Chimera than using FSC measurements in combination with rescaling of the maps. Therefore, the pixel size and range determined by the script serve as a starting point and boundaries for the next fitting in Chimera. Any pixel values within the calculated range can be used to fit the maps in Chimera until the cross-correlation is optimized ('Fit to Map') [Fig. 3(a)]. The cross-correlation of the initial and final maps can also be plotted using FSC in RELION:

relion_image_handler --i mapl.mrc --fsc map2_rescaled.mrc--angpix $1.40>$ fsc.star

The correlation between the maps increases when an optimal scaling factor is found [Fig. 3(b)]. In our particular example, the relative pixel size was off by approximately $6 \%$ (actual, $1.28 \AA$ per pixel; nominal, $1.36 \AA$ per pixel) and the calculated scaling factor was 0.914 (actual, $1.28 \AA$ per pixel; reference, 1.40 Å per pixel).

It should be noted that to perform this cross-correlation in RELION successfully, the origin of the maps to be compared needs to be the same. The reason is that after rescaling, the origin of the rescaled map will be shifted. This can be adjusted using the Chimera 'Fit in map' option in combination with the vop resample command. Firstly, the resampled map needs to be superposed as described above to fit its coordinates to those of the map with which it should be compared. Secondly, by using the vop resample command, a new map with the new coordinates can be saved (vop resample \#0 on Grid $\# 1$, where \#0 is map2_rescaled.mrc and \#1 is map1.mrc) and used for FSC calculations.

\subsection{Merging data sets}

There are different methods of combining cryo-EM data sets with different pixel sizes. One can rescale the original micrographs (Method 1) or rescale the extracted particles (Method 2) (Figs. 1 and 2). Each has specific advantages and disadvantages in handling. For instance, Method 1 involves reprocessing of the data sets after rescaling the micrographs,
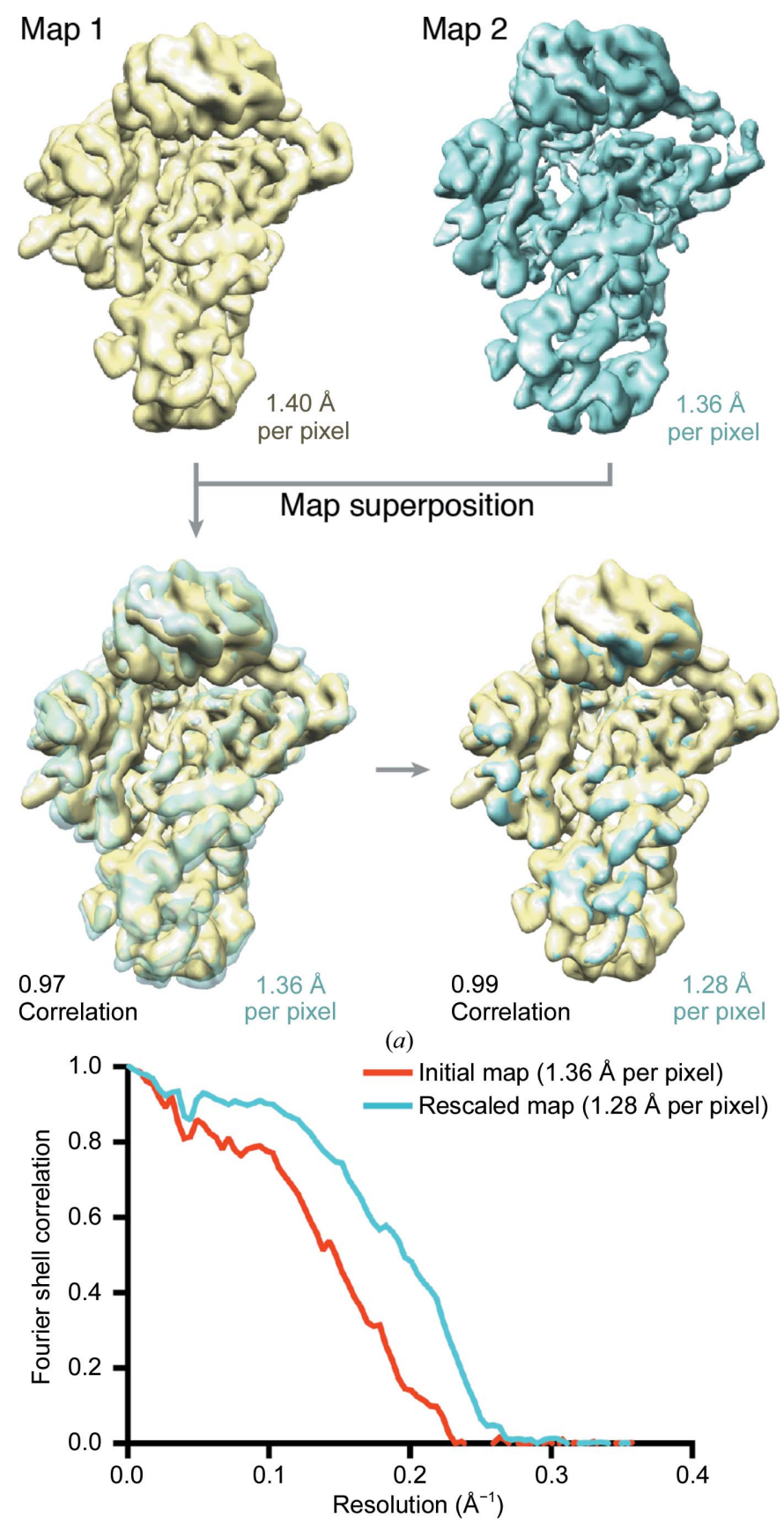

(b)

Figure 3

Scaling factor between data sets. (a) Independent 3D maps obtained from data set I (yellow) and data set II (cyan) vary in volume size owing to differences in the nominal pixel sizes. Superposition of such maps in Chimera shows that correlation between the reconstructions improves when the pixel size of map 2 (cyan) is scaled to fit the pixel size of map 1 (yellow). (b) Fourier shell correlation (FSC) between maps 1 and 2, before and after scaling. The correlation increases at $1.28 \AA$ per pixel. 
while in Method 2 rescaling is performed at the level of particles and the user does not have to reprocess raw movies. On the other hand, Method 1 requires less manipulation of the files after merging when compared with Method 2. In either case, the final result should be similar. For both methods, since rare $2 \mathrm{D}$ views might go missing with extensive $2 \mathrm{D}$ classification before combining the data, it is recommended to merge unclassified particles.

2.3.1. Method 1. In this example, with the same CPF data sets as described above, large-scale data acquisition (4227 movies) was required to overcome preferred orientation (Supplementary Fig. S1). Because microscope time was limited, we opted to collect data using independent microscopes. Initial data processing in RELION 2.0 (FernandezLeiro \& Scheres, 2017) yielded 3D maps to a resolution of approximately $4.00 \AA$ from each microscope. The published structure (EMDB-3908) was obtained by combining 333550 particles and 126617 particles from data set I and data set II, respectively. After merging and further processing of the data, we obtained a final 3D reconstruction at $3.50 \AA$ resolution with a total of 77917 particles (17\% of the total particles), with a contribution of 40518 particles from data set I (3.73 $\AA$ ) and 37399 particles from data set II (3.61 $⿱$ ) ) (Fig. 4 and Supplementary Fig. S1).

(i) Rescale micrographs. The original micrographs can be rescaled using relion_image_handler. To do this, one can use the previously determined relative pixel size (1.28 ̊ per pixel) to rescale the micrographs of the data set II to the final required pixel size (1.40 A per pixel). Type in the command line:
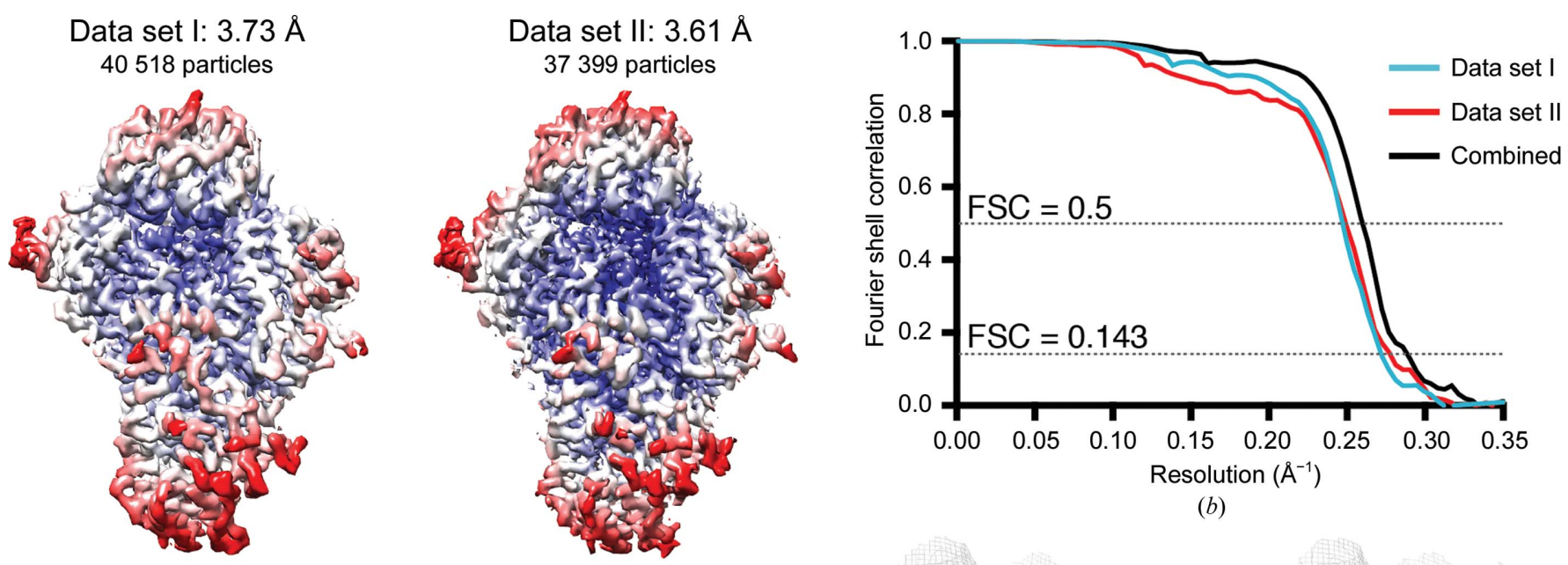

(b)
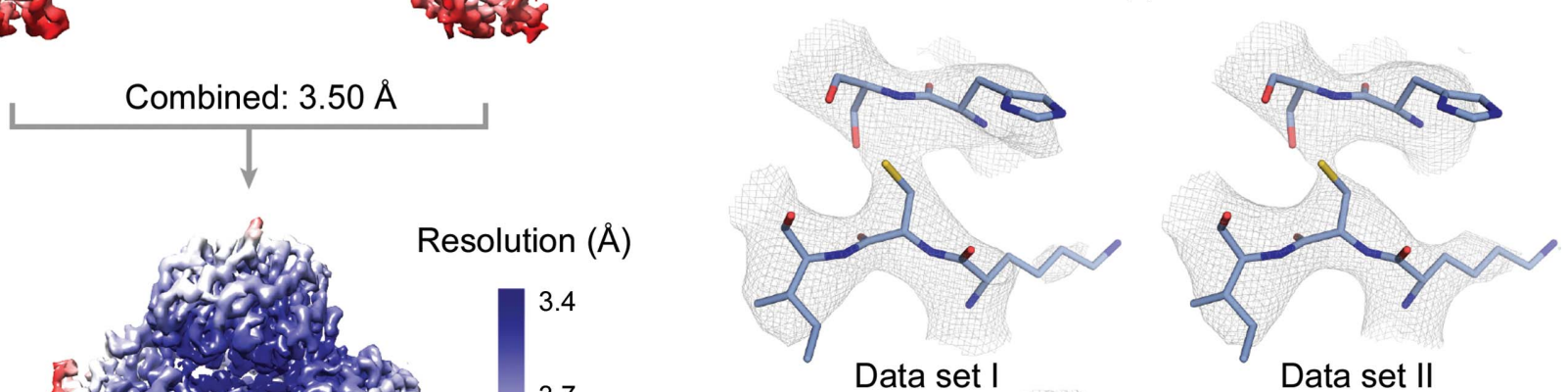

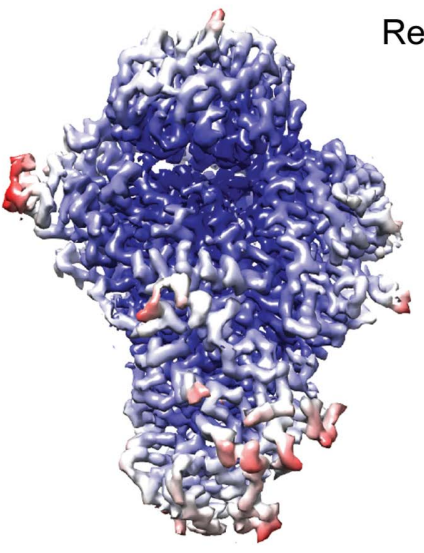

(a)

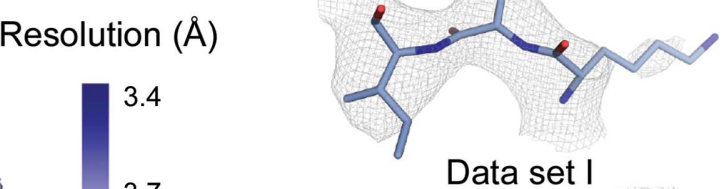

3.7

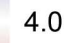

4.3

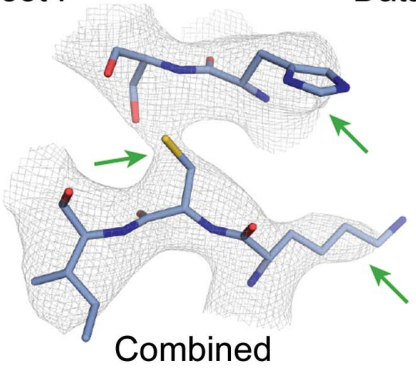

(c)

Figure 4

Merging data sets of the polymerase module of CPF from yeast using Method 1. (a) Reconstructions of the polymerase module of CPF are shown before and after joining the data. The global and local resolution of the final 3D structure (EMDB-3908) improves after combining the data sets. Maps from data sets I and II represent the particle contribution of each data set to the final structure. Local resolution was determined using RELION for 3D reconstructions of particles from data sets I and II alone (at the final/scaled pixel size, i.e. $1.40 \AA$ per pixel) and from combining data sets after scaling data set II using a relative pixel size of $1.28 \AA$ per pixel. Resolutions are given according to the gold-standard criteria. (b) Fourier shell correlation plots for gold-standard refinements. (c) Example density for data sets I and II and combined data sets. The final 3D structure shows finer details in the main chain and side chains when compared with the individual data sets, as indicated by green arrows. 
relion_image_handler--iold_mics_dataset $2 . m r c$ --orescaled_mics_dataset2.mrc--angpix 1.28 -rescale angpix 1.40

Rescaling can be performed on average micrographs (.mrc) and movies (.mrcs). Note that to perform a later polishing step (recommended), it is necessary to rescale the movies. The advantage of rescaling both average micrographs and movies is that the motion-correction step, which is computationally intensive, does not need to be performed again.

(ii) Rerun CTF estimation. CTF parameters need to be recalculated for the scaled micrographs using their new true pixel size (1.40 $\AA$ per pixel). Inaccuracies in the nominal pixel size affect both defocus and contrast transfer function (CTF) estimations (Zhu et al., 1997). As the CTF causes resolutiondependent amplitude modulations and phase reversals in the image, an accurate estimate of CTF parameters is essential to determine 3D structures, particularly at high resolution, when phase shifts become more relevant (Mindell \& Grigorieff, 2003; Cheng et al., 2015). Running CTF estimation on the newly rescaled data set is computationally cheap, and it will guarantee the accuracy of the CTF. After this step, the data sets can be treated as if they had been recorded on the same microscope with the same magnification and pixel size. Particle picking, extraction, 2D classification and further data processing can be performed as before. There is no need to calculate a new box size, and data sets can be merged using the 'Join STAR files' job in RELION (Fig. 2; Fernandez-Leiro \& Scheres, 2017).

(iii) Saving time by rescaling coordinates of previously picked particles. One can save processing or manual picking time by rescaling the coordinates of the already picked or extracted particles from the initial processing. The coordinates will have changed owing to the rescaling of the micrographs. In addition, the magnification and the path to the micrographs will need to be updated in the STAR file. We provide a second Python script called rescale_particles.py (Supplementary Script 2) that takes several columns from a particles.star/data.star file and corrects them based on the pixel sizes. The columns that it uses are _rlnMicrographName, _rlncoordinatex, _rlncoordinatey, _rlnoriginX, _rlnoriginy, _rlnMagnification and _rlnDetectorPixelsize. The inputs for the script are the nominal pixel size used for the $R E L I O N$ run, the relative pixel size calculated and the target/ rescaled pixel size. It also allows the name of the micrographs

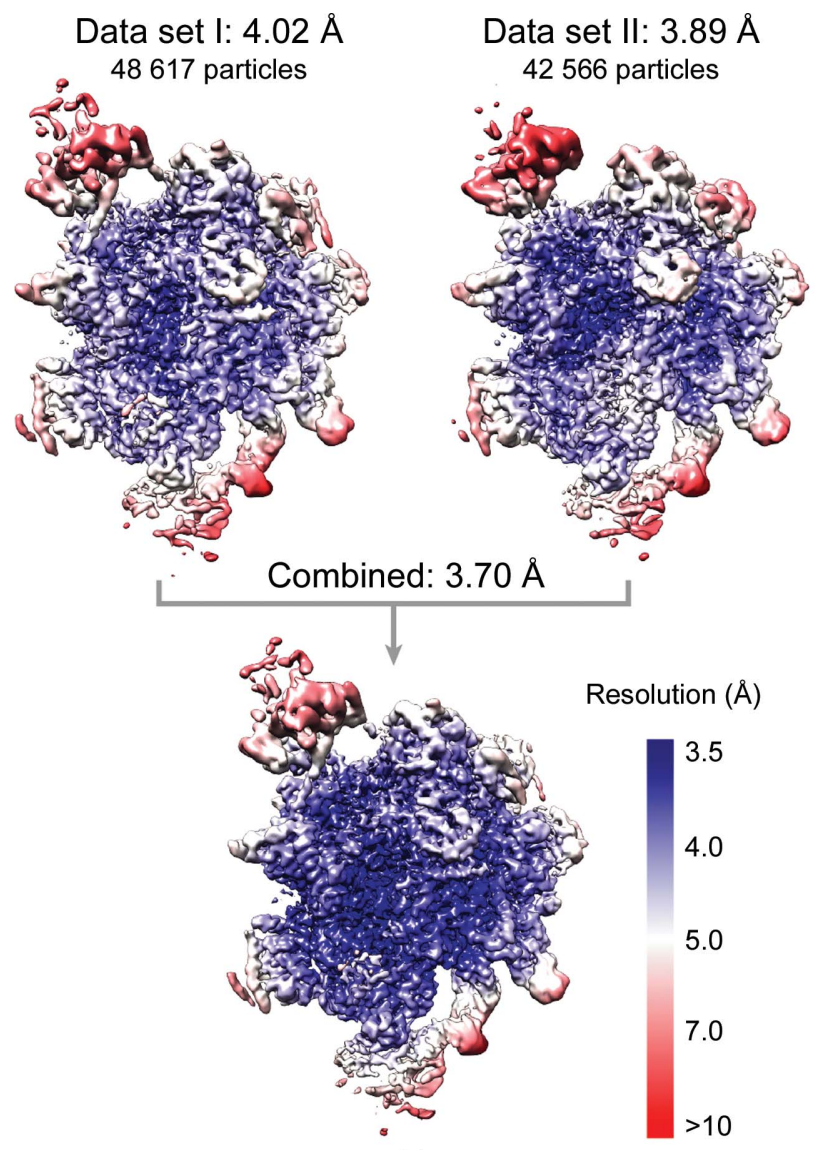

(a)
Data set I: $4.02 \AA$

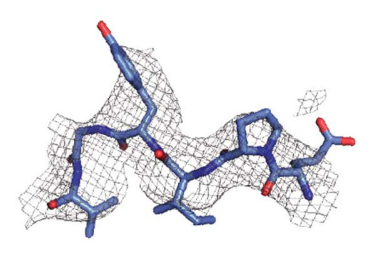

Data set II: $3.89 \AA$

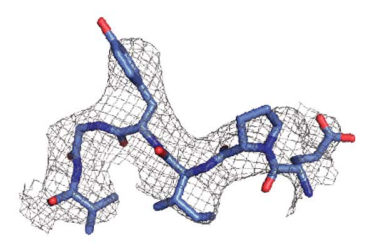

Combined: $3.70 \AA$

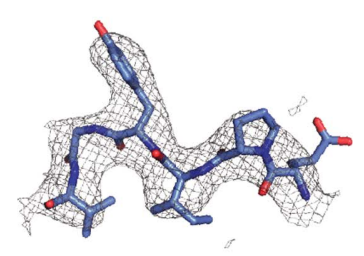

(b)

Figure 5

Merging data sets of the yeast post-catalytic (P complex) spliceosome using Method 2. (a) The global and local resolution of the final 3D structure improves after combining the data sets. Local resolution was determined using RELION for 3D reconstructions of particles from data sets I and II alone (at the final/scaled pixel size) and from combining data sets after scaling data set II using a relative pixel size of $0.880 \AA$ A per pixel. Resolutions are given according to the gold-standard criteria. (b) Example density for data sets I and II and combined data sets. 


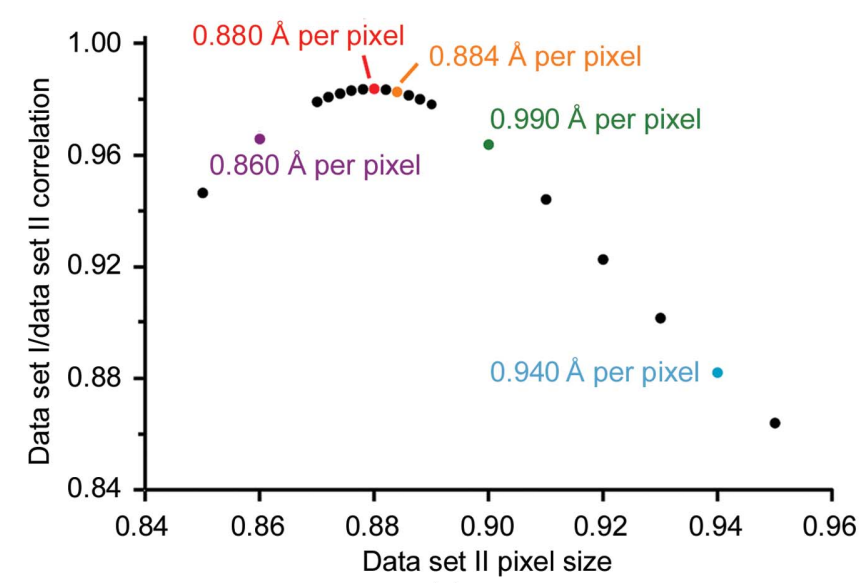

(a)

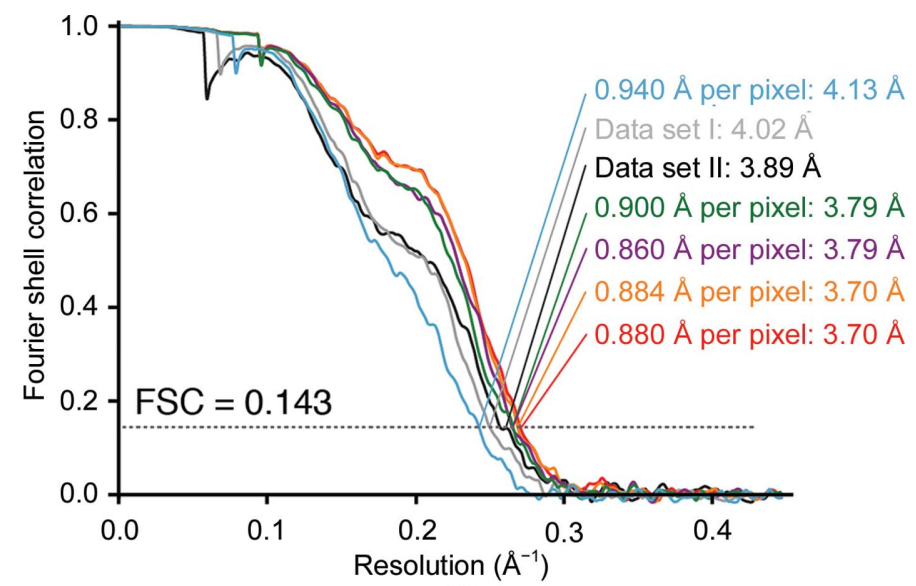

(b)

Figure 6

Effect of scaling. (a) Correlation between the 3D reconstructions calculated from data set I and data set II (at $1.120 \AA$ per pixel) using various pixel sizes for data set II. The correlation was calculated using Chimera as described in the text. (b) Gold-standard FSC for spliceosome reconstructions for data set I alone (light grey), data set II alone (black) and from combining data as described in the text (red). Additional curves come from mis-scaling data set II using the indicated pixel size to $1.120 \AA$ per pixel, then merging with data set I, refining and post-processing. These curves are coloured the same as the corresponding points in $(a)$. Resolutions are given according to the gold-standard criteria.

in the STAR file to be changed (e.g. a new path where the rescaled MRC files are located). The output file is ready to be imported into RELION ('Import') and can be readily used.

python rescale_particles.py --i dataset2.star --o dataset2_rescale.star --pix_nominal 1.36 --pix_relative 1.28 -pix_target 1.40 --mrc_ name_path MRCS_dataset2/

Using this methodology, we successfully combined data sets of the $200 \mathrm{kDa}$ polymerase module of CPF and obtained an improved 3D reconstruction at $3.50 \AA$ resolution. The final map shows an overall improvement in local resolution and density quality (Fig. 4; Casañal et al., 2017).

2.3.2. Method 2. Method 2 differs from Method 1 by not scaling micrographs, but rather scaling particles during extraction from the micrographs (Figs. 1 and 2). This method is demonstrated using the structure of the yeast post-catalytic spliceosome captured immediately after the exon-ligation reaction (Wilkinson et al., 2017). The published structure (EMDB-3979) was obtained from a single data set of 48617 particles collected at $1.120 \AA$ per pixel to $3.73 \AA$ resolution. Subsequently, a second data set of 42566 particles was collected at $0.900 \AA$ per pixel, which refined to $3.89 \AA$ resolution (Supplementary Figs. S1 and S2). Both data sets were collected on Krios 1 at MRC-LMB. The original data set (larger pixel size) is referred to as 'data set I', and in this example unpolished particles were used, which refined to $4.02 \AA$ resolution (Fig. 5). The relative pixel size of data set II was obtained using Chimera, as detailed above, which produced a clear peak of cross-correlation to data set $\mathrm{I}$ at 0.880 A per pixel (Fig. 6).

(i) Re-calculate CTF defocus values. In the example here, data set II was originally processed (including CTF estimation) using a pixel size of $0.900 \AA$ per pixel, which was the reported nominal pixel size at the magnification of data collection. After the determination of the relative pixel size
$(0.880 \AA)$, the CTF parameters need to be re-estimated considering the new pixel size. Alternatively, since to a good approximation the fitted defocus values will depend on the square of the pixel size, one could just multiply these values by $(0.880 / 0.900)^{2}$ :

awk ${ }^{\prime} \mathrm{NF}<5$ \{print $\left.\$ 0\right\} ; / \mathrm{mrc} /\{\$ 3=\$ 3 *(0.880 /$ $0.900) * * 2 ; \$ 4=\$ 4 *(0.880 / 0.900) * * 2 ; \quad \$ 10=10000 ;$ $\$ 11=0.880$ print $\$ 0\}^{\prime}$ micrographs_ctf.star > micrographs_ctf_newapix.star

In this example, $\$ 3$ and $\$ 4$ refer to columns 3 and 4 of the STAR file, which usually correspond to _rlnDefocusU and _rlnDefocusV, $\$ 10$ and $\$ 11$ refer to columns 10 and 11 , corresponding to_rlnMagnification and_rlnDetector PixelSize, and $0.880 / 0.900$ needs to be substituted for the correct ratio between the relative (new) pixel size and the nominal (old) pixel size. The correct column numbers can be found by looking at the first few lines of the RELION STAR file. Something similar can also be achieved with the supplied bash script scale_ctf.sh (Supplementary Script 3), which additionally applies a small constant correction to somewhat account for higher order terms in the CTF equations. It is run as follows (where bold indicates user input):

scale_ctf.sh micrographs_ctf.star

Starting apix: 0.900

New apix: $\mathbf{0 . 8 8 0}$

Read spherical aberration as 27000000 A from star-file header

Acceleration voltage read as 300, will use electron wavelength of $0.0197 \mathrm{~A}$

Defocus scaled by 0.956049 plus a constant correction of -14.602555

Wrote out micrographs_ctf_newapix.star

Besides the defocus values, errors in the pixel size also impact the spherical aberration (Cs) term. While the defocus is 
directly proportional to the square of the wavenumber, the Cs is proportional to the fourth power of the wavenumber. Therefore, the defocus term dominates at lower resolutions, but at higher resolutions the Cs becomes significant. The Cs can be scaled [e.g. for a Cs of $2.7 \mathrm{~mm}$, the new Cs is $2.7 \times$ $\left.(0.900 / 0.880)^{4}\right]$ and modified in the STAR file. With CtfRefine one can keep Cs as it is and refine only defocus values. The fit then becomes accurate.

(ii) Re-extract and scale particles. In RELION a convenient way to scale particles is during the 'Particle Extraction' job type. In this job, one has the option of extracting at a particular box size and scaling this box to another box size. (If one no longer has the micrographs on disk or wishes to scale polished particles, one can also scale extracted particles using relion preprocess). A restriction is that the box sizes must be evennumbered. In the example given, data set II is rescaled from $0.880 \AA$ A per pixel to $1.120 \AA$ per pixel and has a final box size of 420 pixels (the box size of data set I). To scale straight to a box of 420 pixels, one would have to extract with a box size of $420 \times 1.120 / 0.880=534.5$ pixels, which is not possible. A possibility would be to extract with a box of 534 pixels and scale to 420 pixels, giving a scaled pixel size of $534 / 420 \times 0.880$ $=1.119$, which might be accurate enough (see below). Alternatively, we provide a Python script boxscaler.py (Supplementary Script 4), which can find a pair of evennumbered box sizes very close to the desired ratio. It is run as follows (bold indicates user input):

\section{boxscaler.py}

What is the smallest box size to search from? 420 What is the largest box size to search to? 600

What is your starting pixel size? $\mathbf{0 . 8 8 0}$

and how many answers do you want? 1

Start with a 560 pixel box, scale to a 440 pixel box

This will give a scaling factor of 0.78571 , compared to a desired pixel size ratio of 0.78571 , giving a 0.000 per cent error.

This script shows that extracting at box size 560 and scaling to box size 440 will give the desired scaling factor to $0 \%$ error. One can perform this in RELION by creating a 'Particle extraction' job using the run_data.star file generated from refinement of data set II to provide the particle coordinates ('Refined particles STAR file' field) and using the micrographs_ctf_newapix.star file generated in step 1 of Method 2 to provide the micrographs with the correct CTF parameters ('micrograph STAR file' field). 'Particle box size' need to be specified as 560 pixels, 'Rescale particles' as 'Yes' and 'Re-scaled size' as 440 pixels. CRITICAL: from RELION v.3 onwards (Zivanov et al., 2018), if a run data.star file is used for extraction, CTF parameters are by default taken from this file. Add the --use_ctf_in_mic flag to 'Additional arguments' to make sure that CTF parameters are taken from micrographs_ctf_newapix. star. Manually specify the diameter of the background circle for particle normalization as if we were extracting with a box size of 420 . For example, the default diameter is $75 \%$ of the box size before scaling. Here, the new diameter of the background circle should be 400 pixels $=0.75 \times 420 \times 1.120 / 0.880$.

(iii) Crop particles to the correct box size. Particles are now on the correct scale but with a box size of 440 (data set II), and need to be merged with particles with a box size of 420 (data set I). One can crop to a box size of 420 using relion preprocess as follows:

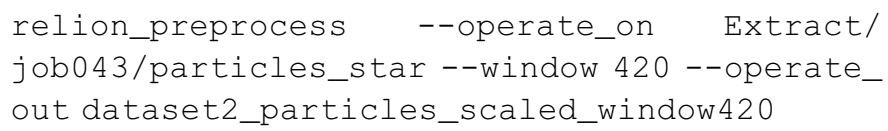

This will write out a stack of particles dataset2_ particles_scaled_window420.mrcs and a corresponding STAR file dataset2_particles_scaled_ window420.star. This STAR file can now be merged with the data set I STAR file using the 'Join STAR files' job in RELION.

In this example, combining the data sets gave a reconstruction at $3.70 \AA$ resolution, with significant improvement in local resolution and density quality compared with either data set reconstructed individually (Fig. 5). After further processing using CTF refinement and Bayesian polishing in RELION 3.0 (Zivanov et al., 2018), we were able to improve the resolution to $3.30 \AA$, a significant improvement over the published reconstruction (Supplementary Fig. S2).

Note: to integrate this method with Bayesian polishing, perform polishing on data set II supplying the following additional flags under 'additional arguments': --window 560 --scale 440

As a result, polished particles will be produced at the correct size but with a box size of 440 . These can be cropped as above as follows:

relion_preprocess --operate_on Polish/job043/ shiny.star--window 420 --operate_out dataset2_ particles_shiny_scaled

The output will be a particle stack dataset2_ particles_shiny_scaled.mrcs and a STAR file dataset2_particles_shiny_scaled.star, which can be joined with data set I.

\section{Effect of pixel size on resolution}

For several reasons, it may not be possible to precisely calculate the correct scaling factor. For example, the resolution of the individual maps may not be high enough for a discrete peak of correlation to emerge when aligning maps in Chimera, or a convenient ratio of box sizes that gives the correct scaling factor may not be available. We tested how accurately the scaling factor needs to be determined (Fig. 6). We scaled data set II in the spliceosome example using different starting pixel sizes: the correct $0.880 \AA$ per pixel, the almost correct $0.884 \AA$ per pixel, the incorrect $0.940 \AA$ per pixel and two close sizes of 0.860 and $0.900 \AA$ per pixel [Fig. 6(a)]. For each case we recalculated the CTF parameters using Gctf (Zhang, 2016) and extracted with a 560-pixel box, scaled to various box sizes (e.g. 440 pixels for $0.880 \AA$ per 
pixel, 470 pixels for $0.940 \AA$ per pixel) and cropped to 420 pixels. After refinement, we determined the resolution using the same mask [Fig. 6(b)]. We found that using $0.884 \AA$ per pixel gave a reconstruction that was almost identical to the correct $0.880 \AA$ A per pixel. Either 0.860 or $0.900 \AA$ per pixel gave only a small reduction in resolution, while $0.940 \AA$ per pixel gave a reconstruction with worse resolution than either of the starting data sets alone [Fig. 6(b)]. This analysis shows that the resolution obtained after reconstruction from merged data sets is relatively tolerant to scaling-factor error, at least in the $3.70 \AA$ resolution range for a $1-2 \mathrm{MDa}$ particle: $0.5-2 \%$ error is acceptable, while 7\% error is not. In principle, higher accuracy should be required when analysing a larger complex or a higher resolution structure (although both of these factors should also facilitate more accurate scaling-factor determination).

\section{Conclusion}

In cryo-EM, protein samples often require extensive biochemical optimization, including sample preparation and vitrification. To obtain structural information that helps to gain insight into specific biological problems, researchers often acquire large data sets or collect data from different preparations. Merging data sets recorded at different microscopes, or with varying conditions of imaging, therefore becomes an important task. In this report, we describe two methods (scaling micrographs or particles) to combine cryoEM data collected at different pixel sizes successfully. We have also shown how errors in pixel-size determination correlate with resolution and provide scripts for the accurate determination of pixel sizes. In our examples, data sets from the same type of microscope (Titan Krios) and detector (K2 equipped with a GIF energy filter) have been merged, improving the resolution. This methodology can be further extended to other cases in which cryo-EM data sets have been acquired with different types of microscopes and detectors. When combining data collected using different detectors, each detector will have a specific MTF file. Different MTF files are likely to have a minimal effect in the final 3D reconstruction, but further analysis is necessary to report their impact at near-Nyquist resolution. It should be noted that adding more data to existing data sets is not always a means to improve the quality of 3D reconstructions. For example, when data quality limits resolution, additional particles will have a marginal effect. To decide whether further data collection is useful, a good strategy is to merge the existing data sets one by one (starting with the best). This will determine whether combining more particles of similar quality improves the quality of the 3D reconstruction. To estimate the number of particles (of similar quality) required to increase resolution one can use Rosenthal and Henderson plots (Rosenthal \& Henderson, 2003; Zivanov et al., 2018). In our examples, the addition of about $50 \%$ more particles helped to increase the resolution of the 3D reconstructions from 3.61 to $3.50 \AA$ for the polymerase module of $\mathrm{CPF}$, and the addition of $100 \%$ more particles increased the resolution from 3.89 to $3.70 \AA$ for the post-catalytic ( $\mathrm{P}$ complex) spliceosome. Importantly, improving the quality of the data will also improve the resolution of the resulting 3D reconstruction (Naydenova \& Russo, 2017). For example, the correction of beam tilt using the new tools in RELION 3.0 (Zivanov et al., 2018) improves the resolution of the polymerase module from data set I to a greater extent than merging with data set II.

We expect that merging data sets will become more relevant in the future as more challenging cryo-EM projects are tackled where one data-collection session is not enough. This work contributes to making merging data sets a routine job.

\section{Related literature}

The following references are cited in the supporting information for this article: Scheres (2014), Zhang (2016) and Zheng et al. (2017).

\section{Acknowledgements}

We thank Thomas G. Martin and Takanori Nakane for help with scripting, Shabih Shakeel for helpful discussions, Lori A. Passmore and Kiyoshi Nagai for supervising work on the cleavage and polyadenylation factor and the spliceosome, and Lori A. Passmore, Sjors H. W. Scheres, Takanori Nakane, Rafael Fernández-Leiro and Andreas Boland for advice and critical reading of the manuscript. We thank Jake Grimmett and Toby Darling for assistance with high-performance computing. Raw micrograph movies and particle coordinates for the polymerase module of $\mathrm{CPF}$ and the $\mathrm{P}$ complex spliceosome data sets have been deposited in the EMPIAR database at EMBL-EBI under accession Nos. EMPIAR-10299 and EMPIAR-10298, respectively. The cryo-EM map of the $3.30 \AA$ resolution reconstruction of $\mathrm{P}$ complex has been deposited in the Electron Microscopy Data Bank under accession No. EMDB-10140. Scripts can be found in the supporting information and in github (https://github.com/ maxewilkinson/CryoEM-scripts).

\section{Funding information}

This work was supported by European Molecular Biology Organization Long-Term Fellowship ALTF66-2015 cofunded by the European Commission (LTFCOFUND2013, GA-2013609409) through Marie Curie Actions (to AC), Gates Cambridge (to AK), a Cambridge-Rutherford Memorial PhD Scholarship (MEW), the European Union's Horizon 2020 research and innovation programme (ERC grant 725685) (to Lori A. Passmore), the Medical Research Council (MC_U105184330 and MC_U105192715) and a European Research Council Advanced Grant (AdG-693087SPLICE3D) (to Kiyoshi Nagai).

\section{References}

Ashtiani, D., Venugopal, H., Belousoff, M., Spicer, B., Mak, J., Neild, A. \& de Marco, A. (2018). J. Struct. Biol. 203, 94-101. 
Battaglia, M., Contarato, D., Denes, P., Doering, D., Giubilato, P., Kim, T. S., Mattiazzo, S., Radmilovic, V. \& Zalusky, S. (2009). Nucl. Instrum. Methods Phys. Res. A, 598, 642-649.

Boland, A., Martin, T. G., Zhang, Z., Yang, J., Bai, X.-C., Chang, L., Scheres, S. H. W. \& Barford, D. (2017). Nature Struct. Mol. Biol. 24, 414-418.

Casañal, A., Kumar, A., Hill, C. H., Easter, A. D., Emsley, P., Degliesposti, G., Gordiyenko, Y., Santhanam, B., Wolf, J., Wiederhold, K., Dornan, G. L., Skehel, M., Robinson, C. V. \& Passmore, L. A. (2017). Science, 358, 1056-1059.

Charenton, C., Wilkinson, M. E. \& Nagai, K. (2019). Science, 364, 362-367.

Chen, J., Noble, A. J., Kang, J. Y. \& Darst, S. A. (2019). J. Struct. Biol. $X ., 100005$.

Cheng, Y., Glaeser, R. M. \& Nogales, E. (2017). Cell, 171, 1229-1231.

Cheng, Y., Grigorieff, N., Penczek, P. A. \& Walz, T. (2015). Cell, 161, 438-449.

Cianfrocco, M. A., Lahiri, I., DiMaio, F. \& Leschziner, A. E. (2018). J. Struct. Biol. 203, 230-235.

Drulyte, I., Johnson, R. M., Hesketh, E. L., Hurdiss, D. L., Scarff, C. A., Porav, S. A., Ranson, N. A., Muench, S. P. \& Thompson, R. F. (2018). Acta Cryst. D74, 560-571.

Faruqi, A. R. \& McMullan, G. (2011). Q. Rev. Biophys. 44, 357390.

Fernández, I. S., Bai, X.-C., Hussain, T., Kelley, A. C., Lorsch, J. R., Ramakrishnan, V. \& Scheres, S. H. W. (2013). Science, 342, 1240585.

Fernandez-Leiro, R. \& Scheres, S. H. W. (2017). Acta Cryst. D73, 496502.

Fica, S. M., Oubridge, C., Wilkinson, M. E., Newman, A. J. \& Nagai, K. (2019). Science, 363, 710-714.

Guo, H., Suzuki, T. \& Rubinstein, J. L. (2019). Elife, 8, e43128.

Harauz, G. \& van Heel, M. (1986). Optik, 73, 146-156.

Kimanius, D., Forsberg, B. O., Scheres, S. H. W. \& Lindahl, E. (2016). Elife, 5, 19.

Kühlbrandt, W. (2014). Science, 343, 1443-1444.

Li, X., Zheng, S. Q., Egami, K., Agard, D. A. \& Cheng, Y. (2013). J. Struct. Biol. 184, 251-260.

Liu, N., Zhang, J., Chen, Y., Liu, C., Zhang, X., Xu, K., Wen, J., Luo, Z., Chen, S., Gao, P., Jia, K., Liu, Z., Peng, H. \& Wang, H. W. (2019). J. Am. Chem. Soc. 141, 4016-4025.

Matzov, D., Aibara, S., Basu, A., Zimmerman, E., Bashan, A., Yap, M.-N. F., Amunts, A. \& Yonath, A. E. (2017). Nature Commun. 8, 723.

McMullan, G., Faruqi, A. R., Clare, D. \& Henderson, R. (2014). Ultramicroscopy, 147, 156-163.

Menny, A., Serna, M., Boyd, C. M., Gardner, S., Joseph, A. P.,
Morgan, B. P., Topf, M., Brooks, N. J. \& Bubeck, D. (2018). Nature Commun. 9, 5316.

Meyerson, J. R., Rao, P., Kumar, J., Chittori, S., Banerjee, S., Pierson, J., Mayer, M. L. \& Subramaniam, S. (2014). Sci. Rep. 4, 7084.

Mindell, J. A. \& Grigorieff, N. (2003). J. Struct. Biol. 142, 334-347.

Naydenova, K. \& Russo, C. J. (2017). Nature Commun. 8, 629.

Noble, A. J., Dandey, V. P., Wei, H., Braschi, J., Chase, J., Acharya, P., Tan, Y. Z., Zhang, Z., Kim, L. Y., Scapin, G., Rapp, M., Eng, E. T., Rice, W. J., Cheng, A., Negro, C. J., Shapiro, L., Kwong, P. D., Jeruzalmi, D., des Georges, A., Potter, C. S. \& Carragher, B. (2018). Elife, 7, e34257.

Noble, A. J., Wei, H., Dandey, V. P., Zhang, Z., Tan, Y. Z., Potter, C. S. \& Carragher, B. (2018). Nature Methods, 15, 793-795.

Nogales, E., Louder, R. K. \& He, Y. (2016). Curr. Opin. Struct. Biol. 40, 120-127.

Pettersen, E. F., Goddard, T. D., Huang, C. C., Couch, G. S., Greenblatt, D. M., Meng, E. C. \& Ferrin, T. E. (2004). J. Comput. Chem. 25, 1605-1612.

Plaschka, C., Lin, P.-C. \& Nagai, K. (2017). Nature (London), 546, 617-621.

Rosenthal, P. B. \& Henderson, R. (2003). J. Mol. Biol. 333, 721745.

Russo, C. J. \& Passmore, L. A. (2014). Science, 346, 1377-1380.

Russo, C. J. \& Passmore, L. A. (2016). Curr. Opin. Struct. Biol. 37, 8189.

Scheres, S. H. W. (2012). J. Struct. Biol. 180, 519-530.

Scheres, S. H. W. (2014). Elife, 3, e03665.

Scheres, S. H. W. \& Carazo, J.-M. (2009). Acta Cryst. D65, 672-678.

Smith, M. T. J. \& Rubinstein, J. L. (2014). Science, 345, 617-619.

Takizawa, Y., Binshtein, E., Erwin, A. L., Pyburn, T. M., Mittendorf, K. F. \& Ohi, M. D. (2017). Protein Sci. 26, 69-81.

Urnavicius, L., Zhang, K., Diamant, A. G., Motz, C., Schlager, M. A., Yu, M., Patel, N. A., Robinson, C. V. \& Carter, A. P. (2015). Science, 347, 1441-1446.

Wade, R. H. (1992). Ultramicroscopy, 46, 145-156.

Wilkinson, M. E., Fica, S. M., Galej, W. P., Norman, C. M., Newman, A. J. \& Nagai, K. (2017). Science, 358, 1283-1288.

Wilkinson, M. E., Lin, P.-C., Plaschka, C. \& Nagai, K. (2018). Annu. Rev. Biophys. 47, 175-199.

Zhang, K. (2016). J. Struct. Biol. 193, 1-12.

Zheng, S. Q., Palovcak, E., Armache, J.-P., Verba, K. A., Cheng, Y. \& Agard, D. A. (2017). Nature Methods, 14, 331-332.

Zhu, J., Penczek, P. A., Schröder, R. \& Frank, J. (1997). J. Struct. Biol. 118, 197-219.

Zivanov, J., Nakane, T., Forsberg, B. O., Kimanius, D., Hagen, W. J., Lindahl, E. \& Scheres, S. H. W. (2018). Elife, 7, 163.

Zivanov, J., Nakane, T. \& Scheres, S. H. W. (2019). IUCrJ, 6, 5-17. 